Acknowledgement: Funding was provided by the Sao Paulo Research Foundation (FAPESP) and Coordination for the Improvement of Higher Education Personnel (CAPES).

Disclosure of Interests: None declared

DOI: 10.1136/annrheumdis-2019-eular.2863

\section{SAT0003 PIM1 REGULATES CD4+ T CELL EFFECTOR FUNCTION IN EARLY RHEUMATOID ARTHRITIS AND HAS POTENTIAL FOR USE AS A NOVEL, MEASURABLE THERAPEUTIC TARGET}

Nicola J. Maney, Amy Anderson, John Isaacs, Arthur Pratt. Newcastle University, Newcastle Upon Tyne, United Kingdom

Background: We previously showed PIM1, among other STAT3-target genes, to be strikingly upregulated in circulating CD4+ $T$ cells of treatment-naïve 'early' rheumatoid arthritis (eRA) patients ${ }^{1,2}$. PIM1 has a recognised role in $T$ cell development, has been implicated in the pathogenesis of autoimmunity ${ }^{3}$, and is the target of specific inhibitors in clinical development in oncology; for example PIM1 was recently identified as a promising target in triple-negative breast tumours ${ }^{4}$.

Objectives: We sought to understand the relevance of dysregulated PIM1 gene transcription for T-cell pathobiology during disease initiation and to determine the effects of its inhibition on $\mathrm{T}$ cell function. We hypothesised that, amongst a readily-identifiable, high PIM1 expressing subgroup of eRA patients, PIM1 overexpression in CD4+ T cells is a targetable early event in pathogenesis that lies downstream of STAT3-mediated IL-6 signalling.

Methods: Peripheral blood was obtained from consenting treatment-naïve arthritis patients or healthy donors and highly pure CD4+ $\mathrm{T}$ cells were isolated. PIM1 knock-down (SMARTpool siRNA, Dharmacon) or protein inhibition (small molecule inhibitors; PIM1-selective TCS-PIM-1 1, Tocris and panPIM AZD1208) was undertaken. Flow cytometric analysis was then used to assess activation and proliferation following CD3/CD28-mediated stimulation.

Results: In eRA, ex vivo CD4+ T cells exhibited an activated, hyper-proliferative phenotype compared with those isolated from healthy donors Significantly higher PIM1 mRNA expression in CD4+ T cells was seen using PrimeFlow (flow cytometry) compared to that of other inflammatory arthritidies. Both PIM1 knock-down, PIM1-specific and panPIM inhibition decreased the activation and proliferation of stimulated eRA (Figure 1A) and healthy donor CD4+ T cells. Moreover, in eRA, PIM1 and panPIM inhibition increased FoxP3 expression (Figure 1B) and increased the proportion of regulatory $\mathrm{T}$ cells (Figure $1 \mathrm{C}$ ).

A

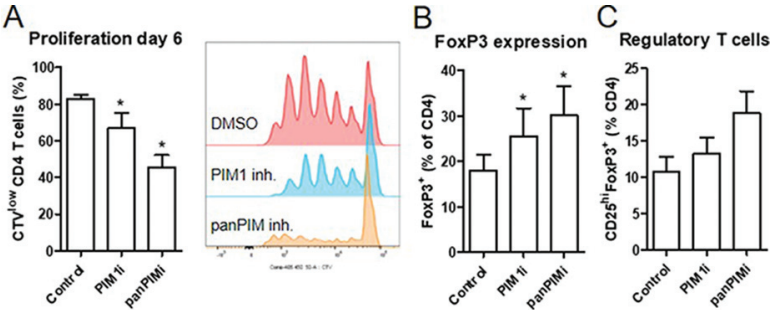

Figure 1. Early RA CD4+ T cell (A) proliferation is significantly decreased by either a PIM1selective or a panPIM small molecule inhibitor, whereas (B) FoxP3 expression and (C) the proportion of regulatory $\mathrm{T}$ cells is increased.

Conclusion: Taken together, these data implicate PIM1 as prominent among genes whose induction may "pre-programme" CD4+ T cells to function aberrantly in disease. Conceivably, targeting PIM1 may prove an attractive approach to regulating aberrant CD4+ $\mathrm{T}$ cell effector function in an identifiable sub-population of early RA patients that exhibit high CD4+ $\mathrm{T}$ cell PIM1 expression in their peripheral blood.

\section{REFERENCES}

[1] Pratt AG et al, Ann Rheum Dis 2012; 71(8) 1374-81

[2] Anderson AE et al, Rheumatology 2018 (in press)

[3] Li Z, et al. J Biol Chem 2014; 289(39):26872-81

[4] Horiuchi D, et al. Nature Medicine 22(11):1321-9

Disclosure of Interests: Nicola J Maney: None declared, Amy Anderson: None declared, John Isaacs Grant/research support from: Pfizer, Grant/ research support from: Pfizer, Consultant for: Abbvie, Pfizer, Roche,
Galvani, Merck, Gilead, Eli Lilly, Amgen, Janssen, Celltrion, NAPP, Consultant for: Abbvie, Pfizer, Roche, Galvani, Merck, Gilead, Eli Lilly, Amgen, Janssen, Celltrion, NAPP, Speakers bureau: Abbvie, Pfizer, El Lilly, Speakers bureau: Abbvie, Pfizer, Eli Lilly, Arthur Pratt Grant/research support from: Dr. Pratt is in receipt of an externally peer-reviewed Investigator Initiated Research grant from Pfizer (£66,000)., Grant/research support from: Dr. Pratt is in receipt of an externally peer-reviewed Investigator Initiated Research grant from Pfizer $(£ 66,000)$., Speakers bureau: Dr Pratt has received honoraria from Eli Lilly and Janssen-Cilag Ltd. for his time in preparing presentations for non-promotional meetings that have been paid directly to Newcastle University., Speakers bureau: Dr Pratt has received honoraria from Eli Lilly and Janssen-Cilag Ltd. for his time in preparing presentations for non-promotional meetings that have been paid directly to Newcastle University.

DOI: 10.1136/annrheumdis-2019-eular.7047

\section{SAT0004 EVALUATION OF AUTOPHAGY IN INFILTRATING AND CIRCULATING LYMPHOCYTES FROM PATIENTS WITH SJOGREN'S SYNDROME}

Serena Colafrancesco ${ }^{1}$, Antonina Minniti ${ }^{1}$, Roberta Priori ${ }^{1}$, Marta Vomero ${ }^{1}$, Cristiana Barbati ${ }^{1}$, Francesca Arienzo ${ }^{1}$, Valentina lannizzotto ${ }^{2}$, Elena Pipi ${ }^{2}$, Joana Campos ${ }^{2}$, Saba Nayar ${ }^{2}$, Francesco Ciccia ${ }^{3}$, Francesca Barone ${ }^{2}$, Guido Valesini ${ }^{1}$, Cristiano Alessandri ${ }^{1} .{ }^{1}$ Sapienza University of Rome, Rome, Italy; ${ }^{2}$ University of Birmingham, Birmingham, United Kingdom; ${ }^{3}$ Università delgli studi della Campania Luigi Vanvitelli, Napoli, Italy

Background: Autophagy is a lysosome mediated catabolic process that promotes cell survival during stress conditions. Aberrant regulation of autophagy is involved in the pathogenesis of a widening spectrum of autoimmune disorders: in particular, activation of autophagy pathway is implicated in aberrant survival of activated $T$ cells. We recently demonstrated that autophagy is up-regulated in CD4+ $T$ lymphocytes from patients with Sjogren's Syndrome (SS) both in peripheral blood and tis sue. Moreover, aberrant autophagy correlates with systemic disease activ ity. Little is known on the role of autophagy in B cells homeostasis in SS

Objectives: To investigate the activation of the autophagy pathway in lymphocytes infiltrating salivary gland in SS and peripheral blood from patients with SS.

Methods: Frozen SS minor salivary glands (MSG) from 20 patients were sectioned and evaluated with immunohistochemistry $(\mathrm{IHC})$ by staining for CD3+, CD20+, and CD21+. MSG sections, sequential to IHC, were stained by Cresyl Violet and microdissected (Laser Capture Microdissection) in order to isolate and collect small infiltrates, large CD21- infiltrates, and large CD21+ infiltrates (GC-like structures). Microdissected GC from frozen human tonsils were used as control. The expression of autophagy genes Atg5 and MAP1LC3II (expressed as $2^{\wedge}$ deltaCT normalized to GADPH) was determined by qPCR on microdissected tissue.

For studies on peripheral blood, 19 SS patients and 11 healthy controls were enrolled, and autophagy, expressed as the ratio between Mean Flu orescence Intensity and the isotopic control (rMFI), was evaluated by Cyto-ID ${ }^{\circledR}$ Autophagy detection kit in T lymphocytes (CD4+ and CD8+) and B lymphocytes (CD19+) using a FACSCalibur cytometer.

Results: Expression of the autophagy genes Atg5 and MAP1LC3 was significantly higher in the GCs from SS salivary glands compared to control tonsils $(p<0.0001$ and $p=0.0013$, respectively). In the salivary glands of SS patients, progressively higher expression levels of both Atg5 and MAP1LC3 mirrored the organization and severity of the inflammatory infiltrates (small vs. large CD21+ infiltrates $p=0.0005$ and $p<0.0001$ respectively).

Immunophenotyping studies revealed activation of the autophagy pathway in both circulating CD19+ and CD4+ lymphocytes from SS ( $p=0.04$ and $\mathrm{p}=0.03$ compared to $\mathrm{HC}$ ); conversely, no pathway activation was detected in CD8+ cells. Of note, in patients with SS activation of autophagy was most marked in circulating CD19+ B cells compared the other subsets [(CD19+ vs CD4+ $(p<0.0001) ; C D 19+$ vs CD8+ $(p=0.001)]$.

Conclusion: Our findings revealed that the autophagy pathway is aberrantly activated in lymphocytes infiltrating salivary glands of SS patients. Of note, progressively higher expression levels of autophagy genes mirrored the severity and extension of inflammatory infiltrates. Immunophenotyping studies revealed that activation of autophagy is preeminent in B cells. Thus, activation of autophagy emerges as a feature of both infiltrating and circulating SS lymphocytes, and as mechanism possibly implicated in the activation and survival of autoreactive cells.

Disclosure of Interests: Serena Colafrancesco: None declared, Antonina Minniti: None declared, Roberta Priori: None declared, Marta Vomero: None declared, cristiana barbati: None declared, Francesca Arienzo: None 
declared, Valentina lannizzotto: None declared, Elena Pipi: None declared, Joana Campos: None declared, Saba Nayar: None declared, Francesco Ciccia Grant/research support from: CELGENE, PFIZER, Consultant for: UCB, NOVARTIS, CELGENE, PFIZER, LILLY, Paid instructor for: UCB, NOVARTIS, CELGENE, PFIZER, LILLY, JANSSEN, Speakers bureau: UCB, NOVARTIS, CELGENE, PFIZER, LILLY, JANSSEN, MSD, ROCHE, AMGEN, Francesca Barone Grant/research support from: GlaxoSmithKline, Roche, UCB Pharma, Actelion, ONO Pharmaceutical, Consultant for: GlaxoSmithKline, Roche, Actelion, ONO Pharmaceutical, Guido Valesini: None declared, cristiano alessandri: None declared

DOI: 10.1136/annrheumdis-2019-eular.5901

\section{SAT0005 DETECTION OF HIGHLY EXPANDED T CELL CLONES IN THE PERIPHERAL BLOOD OF AT RISK INDIVIDUALS FOR RHEUMATOID ARTHRITIS BEFORE THE CLINICAL ONSET OF THE DISEASE}

Céline Lamacchia ${ }^{1}$, Zuleika Calderin ${ }^{1}$, Delphine Courvoisier ${ }^{1}$, Denis Mongin ${ }^{1}$, Stéphane Buhler ${ }^{1}$, Gaby Palmer ${ }^{2}$, Olivia Studer ${ }^{1}$, Cem Gabay ${ }^{1}$, Jean Villard ${ }^{1}$, Axel Finckh ${ }^{1} .{ }^{1}$ University Hospitals of Geneva, Geneva, Switzerland, ${ }^{2}$ University of Geneva School of Medicine, Geneva, Switzerland

Background: Rheumatoid arthritis (RA) is an autoimmune disease with unknown etiopathogenesis. Systemic autoimmunity precedes clinical disease onset, and current evidence suggests that the immune onset of RA takes place outside of the joints several years before clinical manifestations. Expanded $T$ cell clones can be found in the synovial tissue of established RA patients. The mechanisms by which systemic immune abnormalities progress to joint-specific autoimmunity are not yet understood.

Objectives: To examine if expanded $T$ cell clone signatures can be detected in the peripheral blood before the development of clinical RA. Methods: Next-generation sequencing of the T Cell Receptor $\beta$ (TCR $\beta$ ) CDR3 repertoire was performed on genomic DNA isolated from blood samples of individuals genetically at risk for RA, namely first-degree relatives of RA patients (RAFDR) at different pre-clinical phases of disease development (SCREEN-RA cohort), and of matched RA patients used as a control group (SCQM cohort). All individuals were matched for age and sex, and categorized into four groups ( $n=20 /$ group): Group 1: "healthy" asymptomatic RA-FDR without autoantibodies or symptoms associated with possible RA. Group 2: Asymptomatic RA-FDR with evidence of 'systemic autoimmunity associated with RA' defined by high levels of anti-citrullinated peptide antibodies (ACPA; $3 x \geq U L N$ ). Group 3: RA-FDR having presented undifferentiated arthritis $(n=8)$ or having developed classifiable $R A$ after inclusion $(n=12)$. Group 4: patients with established RA of less than 3 years duration. T cell clones were identified by their unique TCR $\beta$ CDR3 sequence. Clones with a frequency over $0.5 \%$ were considered to be highly expanded clones (HEC). Both absolute number and frequency of productive $\mathrm{T}$ cell clones was compared between the 4 groups using mixed effect regression models to account for matching.

Results: As expected, the large majority of clones in the peripheral blood were detected at very low frequency $(<0.1 \%)$ in all groups (Figure $1 \mathrm{~A}$ ). Interestingly, expanded clones $(>0.1 \%$ of total TCR analysed) tended to occur more frequently in later preclinical phases and established disease. A significant difference among groups was observed for highly expanded clones (HEC) $(p=0.001)$. Specifically, the absolute number of HEC was significantly higher in RA patients (group 4; mean 4.65, $\mathrm{p}=0.003$ ) and tended to be higher in symptomatic RA-FDR (group 3; mean 3.4, $\mathrm{p}=0.07$ ) compared to "healthy" RA-FDR (group 1; mean 1.55) (Figure 1B). A trend towards a higher frequency of the top 50 expanded clones was also observed in symptomatic RA-FDR (group 3; mean $0.17 \%$ ) compared to "healthy" RA-FDR (group 1; mean $0.11 \%$ ). At risk individuals defined by the presence of high ACPA levels (group 2) did not differ from "healthy" RA-FDR in terms of absolute number and frequency of clones.

Conclusion: For the first time, highly expanded $\mathrm{T}$ cell clones were detected in the peripheral blood of at risk individuals before the clinical onset of RA, in particular in the later pre-clinical phases of RA development. Tracking these dominant $\mathrm{T}$ cell clones in longitudinal analyses and elucidating their role might help to better understand the earliest pathogenic events in RA.

\section{REFERENCE}

[1] Catrina Al et al. Nat Rev Rheumatol. 2017;13(2):79-86; Klarenbeek PL et al. Ann Rheum Dis. 2012;71(6):1088-93

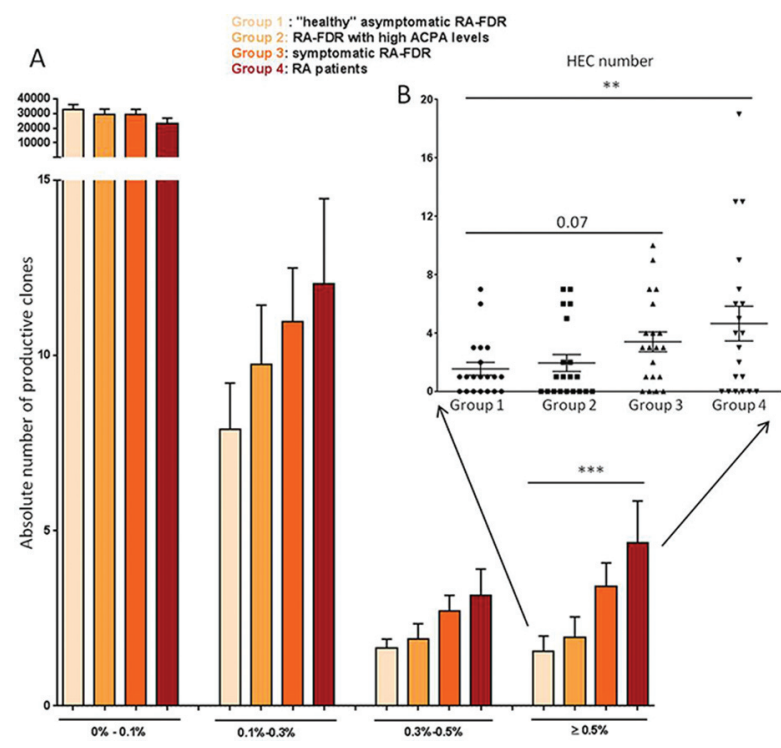

Figure 1. Absolute number of productive TCR clones by clonal size. (A) Bars show mean and standard error of the mean (mixed effect regression model). (B) Each dot represents the HEC number observed for 1 individual (mixed effect regression model).

Disclosure of Interests: Céline Lamacchia: None declared, Zuleika Calderin: None declared, Delphine Courvoisier Grant/research support from: has received an unrestricted grant from MSD for this study, Consultant for: has received consulting fees from BMS, Pfizer, AB2 Bio and Janssen., Paid instructor for: Janssen, Denis Mongin: None declared, Stéphane Buhler: None declared, Gaby Palmer: None declared, Olivia Studer: None declared, Cem Gabay Grant/research support from: Roche, Pfizer, AB2 Bio Ltd, Consultant for: Roche, Pfizer, Lilly, AbbVie, Sanofi, Regeneron, Bristol-Myers Squibb, Novartis, UCB, AB2 Bio Ltd, Debiopharm, Jean Villard: None declared, Axel Finckh Grant/research support from: Bristol-Myers Squibb, Pfizer Inc, Consultant for: AbbVie, A2Bio, Bristol-Myers Squibb, MSD, Roche, Pfizer Inc, and UCB

DOI: 10.1136/annrheumdis-2019-eular.4102

\section{SAT0006 1 T-CELL IMMUNOGLOBULIN AND MUCIN DOMAIN 3 (TIM- 3) IS INCREASED IN ACTIVE RHEUMATOID ARTHRITIS AND ASSOCIATED WITH CLINICAL DISEASE ACTIVITY AND RADIOGRAPHIC PROGRESSION}

Cæcilie Skejø ${ }^{1}$, Morten Aagaard Nielsen ${ }^{1}$, Malene Hvid ${ }^{1,2}$, Aida Solhøj Hansen ${ }^{1}$, Kristian Stengaard-Pedersen ${ }^{2}$, Merete L. Hetland ${ }^{3,4}$, Kim Hørslev-Petersen ${ }^{5}$, Peter Junker ${ }^{6}$, Mikkel Ǿstergaard ${ }^{3,4}$, Stinne Ravn Greisen ${ }^{1}$, Mette Deleuran ${ }^{2,7}$, Bent Deleuran $1,8 .{ }^{1}$ Aarhus University, Biomedicine, Aarhus, Denmark; ${ }^{2}$ Aarhus University, Clinical Medicine, Aarhus, Denmark, ${ }^{3}$ Rigshospitalet, Center for Rheumatology and Spine Diseases, Glostrup, Copenhagen, Denmark, ${ }^{4}$ University of Copenhagen, Clinical Medicine, Copenhagen, Denmark; ${ }^{5}$ University Hospital of Southern Denmark, Danish Hospital for Rheumatic Diseases, Odense, Denmark; ${ }^{6}$ Odense University Hospital, Rheumatology, Odensen, Denmark; ${ }^{7}$ Aarhus University Hospital, Dermato-Venerology, Aarhus, Denmark; ${ }^{8}$ Aarhus University Hospital, Rheumatology, Aarhus, Denmark

Background: Co-inhibitory receptors are important for the regulation of inflammation in autoimmune diseases. Among these, T-cell Immunoglobulin and mucin domain-3 (Tim-3) has recently gained attention, as it is expressed on exhausted T cells co-expressing PD-1 (1).

Objectives: To investigate Tim-3's role in rheumatoid arthritis (RA)

Methods: Early RA (eRA) patients were randomized to conventional methotrexate (MTX) treatment + placebo or MTX + adalimumab (ADA) (2). Plasma were analysed by ELISA at baseline $(n=98)$ and after 3 and 12 months of treatment. Clinical follow up including 28-joint Disease Activity Score with CRP (DAS28CRP) and Total Sharp Score (TSS) were available. Intrasubject differences in sTim-3 between baseline and 3 months were assessed by parametric paired $t$ tests and compared with plasma from HV $(n=44)$ by parametric unpaired $t$ tests. Spearman correlation and Mann-Whitney test were used to investigate relations between sTim-3 and clinical follow up. From chronic RA (cRA) patients $(n=17)$ plasma and synovial fluid were analysed by ELISA and peripheral blood mononuclear cells (PBMC) and synovial fluid mononuclear cells (SFMC) 